\title{
Audit of revised major haemorrhage protocol awareness and equipment levels in a new Glasgow hospital: a rapid improvement event
}

\author{
C. Hughes, K. Owen, R. Ahmed, L. Manchanda and T. McGrattan \\ Anaesthetic Department, Queen Elizabeth University Hospital, Glasgow
}

\section{Background}

The effective management of massive haemorrhage is key capability for any anaesthetic department (1). The British Committee for Standards in Haematology have identified several reasons why hospitals can fail to achieve standards of care, including inadequate training and awareness of local protocols and untimely collection of blood samples and components (2).

An established Major Haemorrhage protocol was revised for use in the new Queen Elizabeth University Hospital theatre department in May 2015. The department was created as part of the merger of three separate hospitals and encompasses 20 theatres.

This audit aimed to assess awareness of the new protocol and appropriate equipment levels in theatres, as part of a rapid improvement event.

\section{Initial Recommendations}

- Staff training on the protocol (already planned)

- Regular checks on the adequate supply of equipment in each theatre by anaesthetic assistants

- Posters displayed in each theatre detailing correct equipment levels

\section{Method}

A baseline snapshot audit was performed in June 2015 .

Areas recorded included:

- Staff in 13 working theatres were asked if they had read the new protocol.

- The number of theatres in which the protocol was visibly displayed was counted.

- The number of theatres with the minimum required levels of equipment (two or more crossmatch request forms, full blood count, urea and electrolyte and coagulation blood bottles) were recorded in 19 theatres.

Results were discussed with the Anaesthetic Sister and recommendations implemented. Three months later an unannounced audit of equipment levels only was undertaken.

\section{Results}

$64 \%(18 / 28)$ of trained nursing staff and 70\% (16/23) anaesthetists had read the protocol. The protocol was displayed in $95 \%$ of anaesthetic rooms and $95 \%$ of theatres (18/19). Theatre equipment levels at baseline and at re-audit are shown in table 1.

Table 1: Results of baseline audit and re-audit. Theatres containing the required amount of equipment expressed in percentage. $n=19$.

\begin{tabular}{|c|c|c|}
\hline Equipment & Baseline (\%) & Re-audit (\%) \\
\hline Crossmatch forms & 32 & 47 \\
\hline Full blood count bottles & 95 & 63 \\
\hline Coagulation bottles & 79 & 84 \\
\hline Crossmatch bottles & 95 & 68 \\
\hline
\end{tabular}

\section{Discussion}

Staff awareness of the new protocol was evaluated in the baseline audit to guide training on the new protocol familiarisation, which was subsequently undertaken. Equipment levels in theatres were variable and unfortunately did not improve after implementation of our initial recommendations.

The British Committee for Standards in Haematology advise that baseline blood samples are taken "at the earliest possible" opportunity (2) requiring the appropriate equipment to be readily available. Therefore, we recommend a Major Haemorrhage trolley, as a source of equipment which can be checked and restocked regularly and rapidly brought to the theatre of the major haemorrhage. This is currently in development.

1. Blood Transfusion and the anaesthetist: Management of Massive Haemorrhage, AAGBI 2010

2. British Committee for Standards in Haematology Writing Group (Stainsby D et al). Guidelines on the management of massive blood loss. Br J Haematol 2006;135:634-641. 